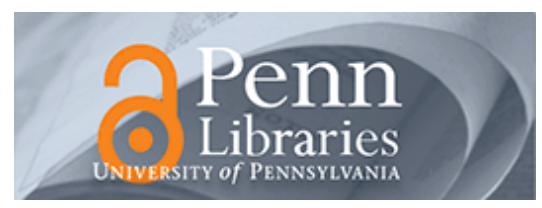

University of Pennsylvania

ScholarlyCommons

4-22-2005

\title{
Colloidal Electroconvection in a Thin Horizontal Cell. I. Microscopic Cooperative Patterns at Low Voltage
}

\author{
Yilong Han \\ University of Pennsylvania \\ David G. Grier \\ New York University
}

Follow this and additional works at: https://repository.upenn.edu/physics_papers

Part of the Physics Commons

\section{Recommended Citation}

Han, Y., \& Grier, D. G. (2005). Colloidal Electroconvection in a Thin Horizontal Cell. I. Microscopic

Cooperative Patterns at Low Voltage. Retrieved from https://repository.upenn.edu/physics_papers/184

Suggested Citation:

Han, Y., Grier, D.G. (2005). Colloidal electroconvection in a thin horizontal cell. I. Microscopic cooperative patterns at low voltage. The Journal of Chemical Physics 122, 164701.

(C) 2005 American Institute of Physics. This article may be downloaded for personal use only. Any other use requires prior permission of the author and the American Institute of Physics. The following article appeared in The Journal of Chemical Physics and may be found at http://dx.doi.org/10.1063/1.1884599.

This paper is posted at ScholarlyCommons. https://repository.upenn.edu/physics_papers/184

For more information, please contact repository@pobox.upenn.edu. 


\title{
Colloidal Electroconvection in a Thin Horizontal Cell. I. Microscopic Cooperative Patterns at Low Voltage
}

\author{
Abstract \\ Applying an electric field to an aqueous colloidal dispersion establishes a complex interplay of forces \\ among the highly mobile simple ions, the more highly charged but less mobile colloidal spheres, and the \\ surrounding water. This interplay can induce a wide variety of visually striking dynamical instabilities even \\ when the applied field is constant. This paper reports on the highly organized patterns that emerge when \\ electrohydrodynamic forces compete with gravity in thin layers of charge-stabilized colloidal spheres \\ subjected to low voltages between parallel-plate electrodes. Depending on the conditions, these spheres \\ can form levitating clusters with morphologies ranging from tumbling clouds to toroidal vortex rings and \\ to writhing labyrinths.

\section{Disciplines} \\ Physical Sciences and Mathematics | Physics

\section{Comments} \\ Suggested Citation: \\ Han, Y., Grier, D.G. (2005). Colloidal electroconvection in a thin horizontal cell. I. Microscopic cooperative \\ patterns at low voltage. The Journal of Chemical Physics 122, 164701. \\ (C) 2005 American Institute of Physics. This article may be downloaded for personal use only. Any other \\ use requires prior permission of the author and the American Institute of Physics. The following article \\ appeared in The Journal of Chemical Physics and may be found at http://dx.doi.org/10.1063/1.1884599.
}




\title{
Colloidal electroconvection in a thin horizontal cell. I. Microscopic cooperative patterns at low voltage
}

\author{
Yilong Han \\ Department of Physics and Astronomy, University of Pennsylvania, Philadelphia, Pennsylvania 19104
}

David G. Grier

Department of Physics and Center for Soft Matter Research, New York University, New York, New York 10003

(Received 9 September 2004; accepted 9 February 2005; published online 22 April 2005)

\begin{abstract}
Applying an electric field to an aqueous colloidal dispersion establishes a complex interplay of forces among the highly mobile simple ions, the more highly charged but less mobile colloidal spheres, and the surrounding water. This interplay can induce a wide variety of visually striking dynamical instabilities even when the applied field is constant. This paper reports on the highly organized patterns that emerge when electrohydrodynamic forces compete with gravity in thin layers of charge-stabilized colloidal spheres subjected to low voltages between parallel-plate electrodes. Depending on the conditions, these spheres can form levitating clusters with morphologies ranging from tumbling clouds to toroidal vortex rings and to writhing labyrinths.
\end{abstract}

(ㄷ) 2005 American Institute of Physics. [DOI: 10.1063/1.1884599]

\section{INTRODUCTION}

Electric fields exert forces on charge-stabilized colloidal particles both directly through their coupling to the particles' charges and also indirectly through their influence on the surrounding electrolyte. Neighboring particles also interact electrostatically and hydrodynamically with each other as they move. The resulting cooperative motions in many-body suspensions can be quite complex, and our understanding of such electrokinetic phenomena remains incomplete despite more than a century of study. ${ }^{1}$

The tendency of colloidal particles to form oriented chains in oscillatory (ac) electric fields was noted nearly a century $\mathrm{ago}^{2}$ and was recognized to result from field-induced dipolar interactions. More recently, ac fields also have been found to organize colloidal spheres into circulating chevron bands oblique to the field, ${ }^{3-7}$ glassy bands perpendicular to the field, ${ }^{8}$ and even highly ordered colloidal crystals. ${ }^{8-10}$ Still other patterns form when electric fields are used to drive charged colloid against electrodes' surfaces, with twodimensional fractal aggregates appeared in monodisperse suspensions ${ }^{11-14}$ and planar superlattices forming in binary colloids. ${ }^{15}$ More recently, a colloidal model system has been introduced whose phase diagram can be tuned with salt concentration and ac electric field. ${ }^{16}$

Even constant (dc) electric fields give rise to complex dynamics. For example, charged spheres driven onto electrodes' surfaces by dc fields self-organize into epitaxial colloidal crystals. ${ }^{17-20} \mathrm{dc}$ fields also have been observed to induce complex labyrinthine patterns in nonaqueous nanocolloids. $^{21}$

Most of these effects have been explained on the basis of electrostatic and hydrodynamic coupling among the spheres mediated by field-induced forces and fluxes of ions. These complex interdependent processes generally are said to result in electrokinetic or electrohydrodynamic forces acting on the particles, with the former typically referring to systems with intrinsic equilibrium surface charges and the latter to intrinsically neutral systems. This synthesis, however, has not provided explanations for phenomena such as the compression of colloidal fluids into three-dimensional crystals by oscillatory electroosmosis. ${ }^{9}$ Indeed, striking and surprising fieldinduced phenomena can arise in what might appear to be the simplest systems.

This paper reports the remarkably diverse set of patterns that charged colloidal particles can form as they sediment under gravity in a vertical dc electric field. Preliminary results have been published in Ref. 22. Beautiful, highly organized dynamic patterns with spatial periods between 20 and $200 \mu \mathrm{m}$ form at biases just above the threshold for electrolysis. These are supplanted by macroscopic patterns with spatial periods extending to millimeters at higher biases. We describe how such patterns form through the interplay of electrohydrodynamic coupling driven by reaction diffusion of ions and the uniform body force provided by gravity. We further show that the microscopic patterns result from manyparticle interactions rather than an underlying convective instability of the electrolyte.

\section{EXPERIMENTAL SYSTEM}

The experimental apparatus is shown schematically in Fig. 1. Our samples consist of aqueous dispersions of silica spheres $3.0 \mu \mathrm{m}$ in diameter (Bangs Laboratories, Lot No. 4181) confined to slit pore between a glass microscope slide and a coverslip. Samples are diluted 30-fold with deionized water from a Barnstead nanopure water polishing system to a typical volume fraction of $0.3 \%$. The glass surfaces are cleaned by etching for several hours in a 3:1 mixture of $\mathrm{H}_{2} \mathrm{SO}_{4}$ and $\mathrm{H}_{2} \mathrm{O}_{2}$, after which they are rinsed thoroughly in running deionized water and dried in air in an oven. The inner glass surfaces are coated by vacuum deposition with 


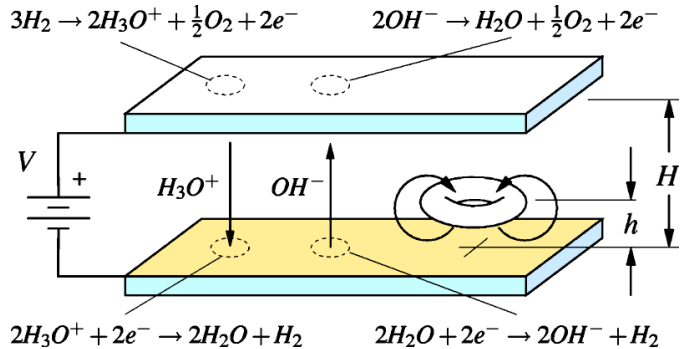

FIG. 1. Parallel-plate electrochemical cell with semitransparent electrodes on the inner glass surfaces. The fluxes of ions are shown schematically together with the electrode reactions responsible for their evolution. A single colloidal vortex ring is shown schematically, levitated to height $h$ in the interelectrode gap $H$. The arrows indicate the vortex ring's observed circulation direction.

$10 \mathrm{~nm}$ thick gold electrodes on $10 \mathrm{~nm}$ thick titanium or chromium wetting layers immediately before assembly. While still optically thin, the electrodes have a resistivity of less than $50 \Omega$ per square and allow us to apply uniform vertical electric fields to the confined suspension. Conductive epoxy is used to make contact to the electrodes once the cell is assembled. In most cases, glass spacers were used to set the electrode separation $H$ in the range $100 \mu \mathrm{m}<H<400 \mu \mathrm{m}$ across the $4 \times 1.5 \mathrm{~cm}^{2}$ observation area. Glass access tubes bonded to holes drilled through the slides were left open to air so that the suspension equilibrates to a $p \mathrm{H}$ of roughly 5.5 through the dissolution of carbon dioxide. Under these conditions, the silica spheres acquire a surface charge density of roughly $-0.4 \mathrm{mC} / \mathrm{m}^{2} .{ }^{23}$ As the density of colloidal silica is roughly $2 \mathrm{~g} / \mathrm{cm}^{3}$, the $3.0 \mu \mathrm{m}$ diameter spheres sediment rapidly onto the lower electrode. Consequently, we use the areal coverage $\phi$ to report the concentration of particles, with $\phi$ $=100 \%$ corresponding to a close-packed monolayer and $\phi$ $=200 \%$ corresponding to a bilayer. Colloidal patterns are imaged with charge-coupled device (CCD) camera through an inverted optical microscope. The images and videos reported in this paper were obtained looking upward through the lower electrode, in the direction opposite to gravity. We define the applied dc voltage to be positive if the upper electrode is positive so that the electric field is directed downward, but the electrostatic forces on negatively charged particles point upward.

The electrochemical processes in a parallel-plate water electrolysis cell are described in most standard references, such as Refs. 24 and 25, and are summarized in Fig. 1. Decomposing a water molecule requires an input of $1.23 \mathrm{eV}$, independent of the salt concentration or the $p \mathrm{H}$ value of the solution. In practice, however, the redox potential is at least $0.6 \mathrm{~V}$ higher due to a voltage drop across the electrodeelectrolyte interface known as the overvoltage (overpotential). Once the threshold for hydrolysis is reached, the electrodes rapidly become coated with insulating layers of $\mathrm{H}_{2}$ and $\mathrm{O}_{2}$. These barriers soon stop the electrode reactions unless the evolved gases form bubbles that escape the electrodes. A substantial nucleation barrier impedes the evolution of $\mathrm{H}_{2}$ and $\mathrm{O}_{2}$ bubbles large enough to expand and escape the electrodes. Consequently, a bias as large as $2.5-3.0 \mathrm{~V}$ typi-

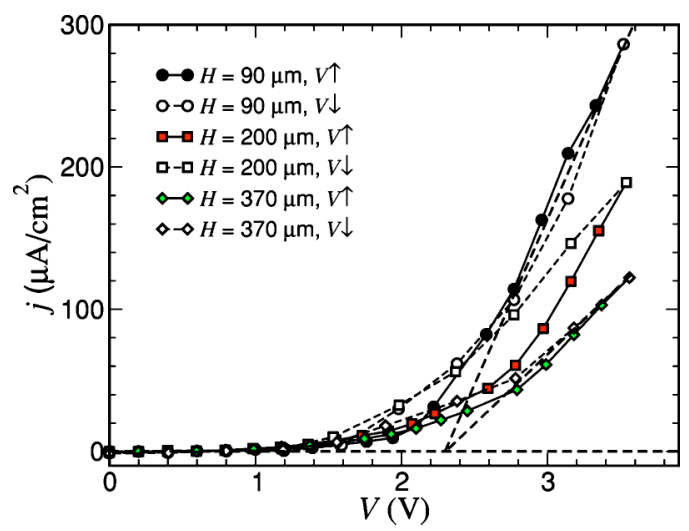

FIG. 2. Current density $j(V)$ as a function of applied voltage in samples of deionized water in three cells of thickness $H=90,200$, and $370 \mu \mathrm{m}$. A single cycle of increasing and decreasing bias is shown for each sample. Values were recorded after 130 s relaxation at each voltage. The intersection of the extrapolated linear trends yields the threshold for hydrolysis.

cally is required to maintain hydrolysis in quasi steady state. The range of threshold biases is due to inevitable variations in the surface condition of the electrodes.

Our sample cells' vacuum-deposited thin film gold electrodes yield threshold biases between 2.6 and $3.2 \mathrm{~V}$. Visible bubbles begin to appear between a minute and an hour after the bias is applied, depending on the voltage. The onset of bubble evolution sets our observation time, and decreases with increasing voltage or salt concentration. Although the redox potential for gold in water is only $1.5 \mathrm{~V}$, the overpotential prevents the anode from etching, so that a negligible concentration of gold ions, $\mathrm{Au}^{3+}$, is released into solution. Indeed, no visible or electrochemical change in the electrodes is seen even after several hours operation at a steady bias. Even in the absence of added salt, the electrolyte in our samples has total ionic concentrations of roughly $n_{\mathrm{HCO}_{3}^{-}} \approx n_{\mathrm{H}_{3} \mathrm{O}^{+}} \approx 3 \mu \mathrm{M}$ and $n_{\mathrm{OH}^{-}} \approx 3 \mathrm{nM}$. A $0.1 \mu \mathrm{m}$ thick Debye-Hückel screening layer forms at each electrode at these concentrations, beyond which the field would not penetrate at equilibrium. During hydrolysis, however, the electrodes act like sources and sinks of $\mathrm{H}_{3} \mathrm{O}^{+}$and $\mathrm{OH}^{-}$ions whose currents establish both an electric field and a $p \mathrm{H}$ gradient through the cell, with the lower half volume becoming acidic and the upper half basic under positive bias.

To quantitatively characterize hydrolysis in our cell, we measured the current density $j(V, t)$ under the simplest conditions: the parallel-plate cell filled with deionized water without colloid. We performed sweeping voltammetry by increasing the applied bias in discrete steps of $0.2 \mathrm{~V}$ from 0 to $3.6 \mathrm{~V}$ and then back down to $0 \mathrm{~V}$, waiting between 2 and 5 min at each voltage level. Figure 2 shows the voltage dependence of the steady-state current in cells of different interelectrode separations $H$. Each curve consists of two linear parts, one at low bias in which little current flows and another ohmic regime at higher biases. Their intersection yields the threshold for water decomposition. ${ }^{25}$ Below the decomposition voltage, the small but nonvanishing current is due to the continuous dissolution of $\mathrm{H}_{2}$ and $\mathrm{O}_{2}$ molecules. This is known as the residual current and also increases with the applied voltage. 


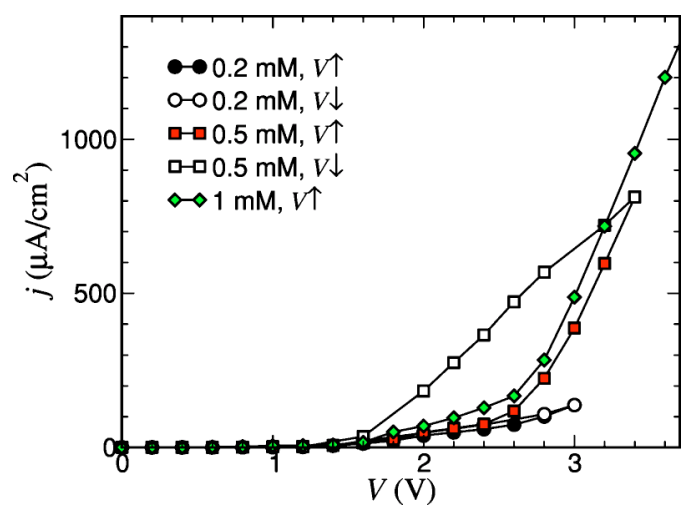

FIG. 3. Current density as a function of applied voltage at different salt concentrations. The samples are aqueous solutions of $\mathrm{NaCl}$ without colloid in a cell $H=200 \mu \mathrm{m}$ thick. The current was measured after $130 \mathrm{~s}$ relaxation at each step in an increasing sequence of applied biases.

The higher conductivity of the high-bias regime is fed by the additional ions from the decomposition of water. Adding $\mathrm{NaCl}$ increases the conductivity but does not affect the threshold, as shown in Fig. 3. For this reason, $\mathrm{NaCl}$ is referred to as indifferent electrolyte.

From the slopes of the data in Fig. 2, we estimate the equilibrium conductivity of our electrolyte to be $\sigma \approx 4$ $\times 10^{-4} \Omega^{-1} \mathrm{~m}^{-1}$. Adding colloidal spheres to the deionized water increases the ionic concentration and thus the conductivity. Adding roughly $\phi=100 \%$ of silica or polystyrene sulfate (PS) colloid increases the conductivity to $7 \times 10^{-4}$ or $20 \times 10^{-4} \Omega^{-1} \mathrm{~m}^{-1}$, respectively. From these values, we estimated the $p \mathrm{H}$ for these systems to be 5.0, 4.7, and 4.2, respectively, with the negatively charged colloidal spheres acting as weak acids.

\section{COLLOIDAL ELECTROHYDRODYNAMIC PATTERN FORMATION}

The field-driven fluxes of ions and charged particles induce flows in the surrounding electrolyte and can give rise to electrohydrodynamic instabilities. These are summarized for one class of systems in the state diagrams in Fig. 4. They consist of two primary categories: patterns formed by clusters of particles levitated collectively into the bulk of the electrolyte and interfacial patterns formed by particles that remain near the electrodes. These two classes can coexist, but typically remain well separated. The following sections describe the various patterns' distinctive characteristics and explain the circumstances under which they form. These diagrams should be considered only semiquantitative because the observed sequence of patterns appears to be very sensitive to parameters such as the local $p \mathrm{H}$ that are not easily controlled externally, and therefore is not precisely reproducible.

\section{A. Interfacial crystals}

The most commonly observed manifestation of electrokinetic interactions among charge-stabilized colloid is the field-induced formation of colloidal crystals on the electrodes. ${ }^{18}$ These form once charged colloidal spheres deposit onto the oppositely charged electrode. Once there, the
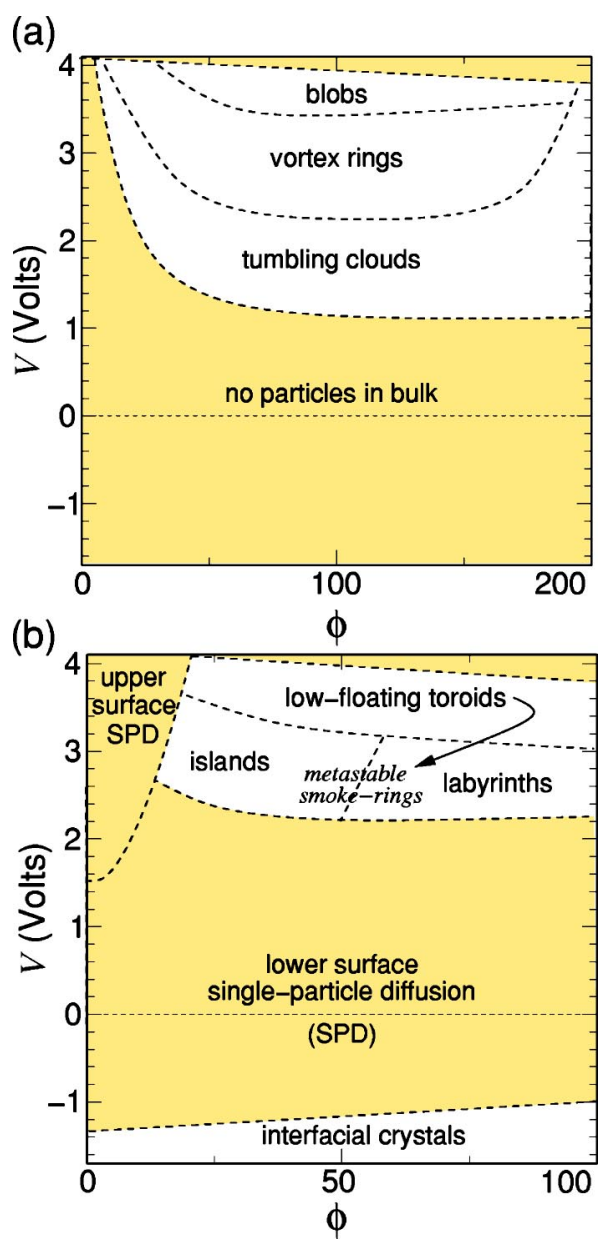

FIG. 4. Overview of field-induced pattern formation for silica spheres $\sigma$ $=3 \mu \mathrm{m}$ in diameter in a slit pore of height $H=200 \mu \mathrm{m}$. Results are presented as a function of applied bias $V$ and initial areal density $\phi$. No patterns form in the shaded regions, and bulk electroconvection supersedes microscopic pattern formation for biases above roughly 4 V. (a) Patterns formed by levitated clusters of particles. (b) Patterns formed by particles near the electrodes.

particles distort the local fluxes of simple ions near the electrodes. The resulting structured flows then mediate strong and long-ranged hydrodynamic attractions among the spheres. ${ }^{18}$ These, in turn, cause the spheres to coalesce into crystalline islands on the electrode.

Such electrohydrodynamically bound crystals also can be induced by an ac field. ${ }^{26}$ At high enough frequencies, however, the dielectric spheres' induced dipole moments mediate an in-plane repulsion strong enough to and the interfacial crystals dissociate destabilize the interfacial crystals. ${ }^{26}$

Field-induced interfacial crystals have been observed in silica spheres, colloidal gold, ${ }^{17}$ highly charged polystyrene sulfate spheres, ${ }^{12,17,19,26}$ and even nominally neutral hydroxyl terminated polystyrene spheres, over a wide range of diameters, and on both indium tin oxide, ${ }^{17,19,26}$ and gold electrodes. The threshold voltage for crystallization can be very low if the spheres' diffusion is slow and if their inherent electrostatic and dipolar repulsions are relatively weak.

In our system, we have observed that the threshold voltage for field-induced crystallization depends on the particles' mobility and charge as shown in Fig. 5, but does not depend on the thickness of the cell. Larger spheres crystallize at 


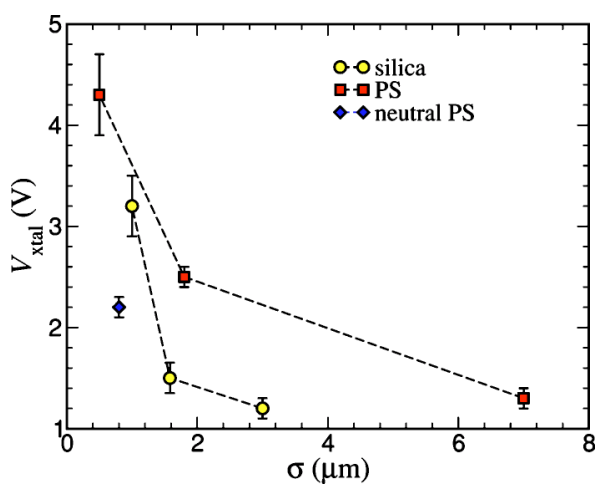

FIG. 5. Threshold voltage for interfacial crystallization for silica, polystyrene sulfate (PS), and neutral polystyrene spheres over a range of particle diameters.

smaller biases both because they are less diffusive and also because the larger distortions they cause in the surrounding ionic fluxes give rise to stronger attractions. The largest silica spheres we studied, with diameters of $3 \mu \mathrm{m}$ crystallized below the $1.23 \mathrm{~V}$ redox potential of water. Weakly charged hydroxyl-terminated polystyrene spheres reproducibly crystallize at the lowest voltages of all, presumably because of their substantially weaker electrostatic repulsions.

\section{B. Colloidal vortex rings}

In this section, we examine some more complex phenomena that arise under a more restricted set of conditions, and which provide new insights into the interplay of electrohydrodynamic and other forces in macroionic systems.

Applying a positive bias in our cell tends to drive negatively charged silica spheres upward into the bulk electrolyte. The field is uniform in our parallel-plate geometry, so that the upward electrophoretic force on spheres of constant charge therefore should be independent of height $h$ in the cell. Under such conditions, spheres dense enough to sediment under gravity should remain near the lower electrode at low biases, and should rise directly to the upper electrode at higher biases where electrohydrodynamics forces exceed gravity.

This effect should be accentuated in charge-regulating silica spheres because the magnitude of their surface charge increases with $p \mathrm{H}$, and therefore should increase with $h$. Indeed, individual isolated silica spheres exhibit hysteresis in their promotion to the upper electrode with increasing bias, and relaxation to the lower electrode with decreasing bias, consistent with these considerations. More concentrated dispersions, on the other hand, exhibit substantially more interesting collective behavior.

At biases between 2.2 and $4 \mathrm{~V}$, which only slightly exceed the threshold for hydrolysis, the background electrolyte is stable against convective instabilities. Nevertheless, initially quiescent collections of spheres develop several classes of striking many-body convective patterns that highlight a previously unsuspected role for cooperativity in chargestabilized dispersions subjected to electric fields. Unlike the electrohydrodynamically stabilized surface crystals de-

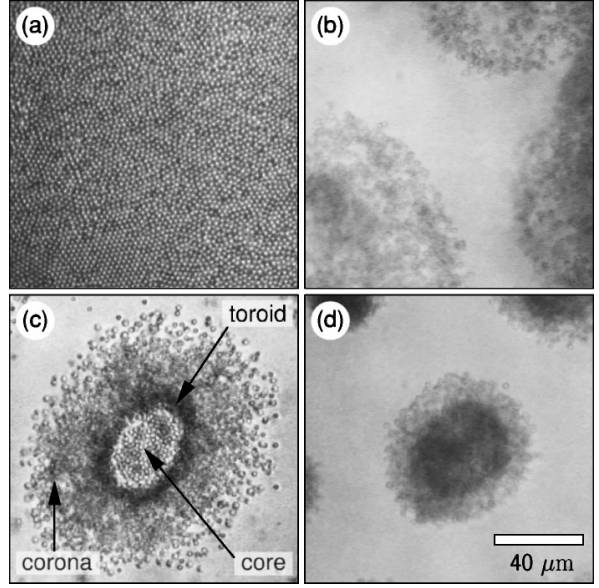

FIG. 6. Typical patterns formed in dispersions of $3.0 \mu \mathrm{m}$ diameter silica spheres at $H=200 \mu \mathrm{m}$. (a) $V=0$, equilibrium monolayer, $\phi \approx 100 \%$. (b) $V$ $=2.6 \mathrm{~V}$, diffuse tumbling clouds. (c) $V=3.0 \mathrm{~V}$, circulating flowerlike cluster levitated to $h=40 \mu \mathrm{m}$. (d) $V=3.8 \mathrm{~V}$, compact circulating cluster, also at $h$ $=40 \mu \mathrm{m}$.

scribed in previous reports, these quasi-steady-state patterns form in the bulk of the electrolyte and reflect the interplay of electrohydrodynamic forces and gravity.

Figure 6 shows the typical sequence of dynamic patterns that forms when an upward bias is applied to dispersions of $3.0 \mu \mathrm{m}$ diameter silica spheres in water. Positively biasing the upper electrode by as much as $0.8 \mathrm{~V}$ causes no discernible out-of-plane motion. Abruptly applying a larger bias in the range $0.8 \mathrm{~V} \lesssim V \lesssim 2.5 \mathrm{~V}$ causes a transient in which spheres jump off the lower surface and then settle back to the bottom. This transient reflects the establishment of static ionic gradients within the water that screen out the field.

The spheres' collective behavior passes through distinct regimes as they are driven out of equilibrium. Just above threshold, the monolayer of spheres breaks into diffuse billowing clouds occupying the lower half of the sample cell, as shown in Fig. 6(b). Pushing the system further from equilibrium might be expected to yield increasingly chaotic behavior; but quite the opposite occurs. Increasing the bias beyond $2.6 \mathrm{~V}$ coalesces the itinerant clouds into extraordinary flowerlike clusters such as the example in Fig. 6(c), all floating with their midplanes at $h=40 \mu \mathrm{m}$ above the lower wall. Each cluster forms around a rapidly circulating toroid whose spheres travel downward along the inner surface and return upward along the outside, completing one cycle in a few seconds. The sense of this circulation is indicated schematically in Fig. 1. Because these clusters' structure and motion are reminiscent of hydrodynamic vortex rings, we will refer to them as colloidal vortex rings.

Colloidal vortex rings formed at higher biases tend to consist of fewer clusters, each containing a larger number of spheres at higher density. The dense yet vigorously circulating cluster in Fig. 6(d) was observed at $V=3.8 \mathrm{~V}$. Increasing the bias once clusters have already formed causes each to shrink and its circulation to accelerate. Such over-driven clusters merge with their neighbors until they achieve the size appropriate to the final bias. The clusters' height, $h$, does not change appreciably with voltage.

By around $V=4 \mathrm{~V}$, some clusters' density increases 


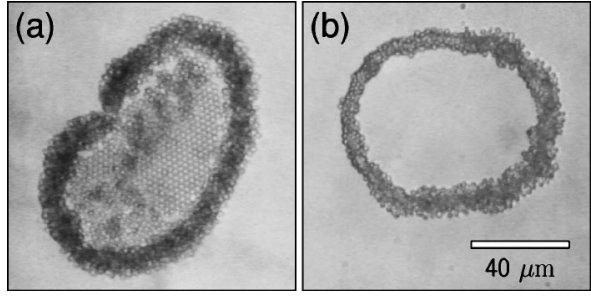

FIG. 7. Bare colloidal vortex ring with and without central core. $3.0 \mu \mathrm{m}$ diameter silica spheres in $H=200 \mu \mathrm{m}$ cell. (a) Compact toroidal cluster enclosing a domain of two-dimensional colloid crystal at $V=2.8 \mathrm{~V}$. The toroid completes one cycle of circulation in about $1.5 \mathrm{~s}$. (b) Compact toroidal cluster with empty core.

enough that they become jammed and stop circulating. These compactified clusters still dissociate immediately once the bias is removed.

At higher voltages, the electrolyte itself becomes unstable against electroconvection. The resulting patterns span the electrochemical cell, with the colloid acting principally as passive tracers. We will discuss this distinct range of conditions elsewhere.

Most often, a densely packed colloidal vortex ring is surrounded by a diffuse circulating corona that extends outward for tens of micrometers. Figure 7 shows alternate forms consisting of compact circulating toroidal clusters without coronas. Such bare colloidal vortex rings form most often at slightly lower voltages, $2.6 \mathrm{~V}$ versus $2.8 \mathrm{~V}$, and can coexist with fully dressed colloidal vortex rings. These clusters also are levitated to $h \approx 40 \mu \mathrm{m}$ in a system of thickness $H$ $=200 \mu \mathrm{m}$.

Although colloidal vortex rings somewhat resemble conventional laminar vortex rings, ${ }^{27}$ they are driven by quite different mechanisms ${ }^{28}$ and also have other distinctive features. Perhaps their most remarkable characteristic is the presence of close-packed colloidal crystals at their centers. The example in Fig. 7(a) is particularly noteworthy because much of its core consists of a static crystalline monolayer. These close-packed spheres are not flocculated, and disperse immediately once the driving field is turned off. Consequently, the crystals' formation and stability suggests the existence of a stagnation plane along each cluster's midplane, quite unlike the streaming flow within a purely hydrodynamic vortex ring.

The stagnation plane must end at the edge of the circulating toroid, and indeed particles from the core sometimes are swept into the circulating flow. While the crystalline domains tend to be stable for the duration of an experimental run (up to $10 \mathrm{~min}$ at $V=3 \mathrm{~V}$ ), some are incorporated into the surrounding toroid after a few minutes, leaving hollow circulating rings such as that in Fig. 7(b).

In most cases, the crystalline monolayer core evolves from a layered fluidlike state as shown in Fig. 8. As the ring's diameter decreases with increasing bias, the concentration of spheres in the core increases to the freezing point, and a static crystal results.

Individual colloidal vortex rings sometimes develop breathing-mode instabilities with periods of a few seconds, with one cycle of a typical structural oscillation appearing in Fig. 9. The usual circulation continues throughout the oscil-
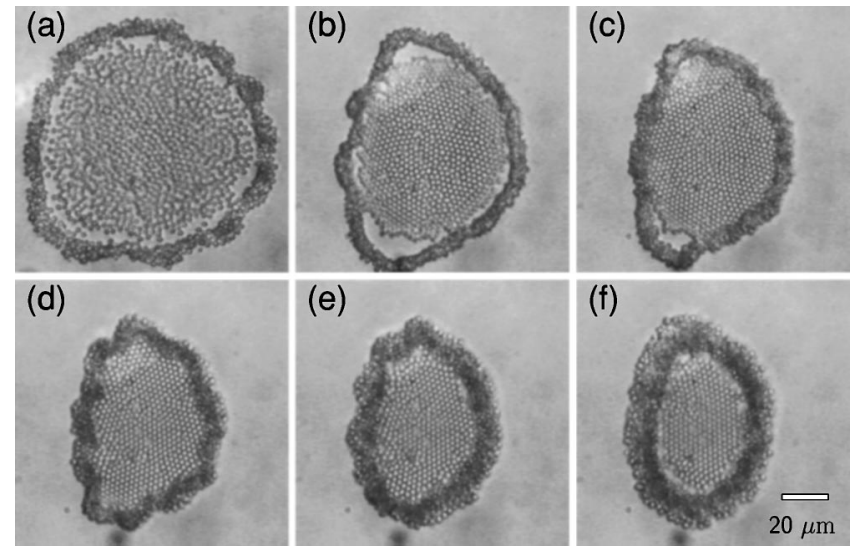

FIG. 8. Central crystal formed by compression in a dispersion of $3.0 \mu \mathrm{m}$ diameter silica spheres in a layer of thickness $H=200 \mu \mathrm{m}$. The sequence of photographs show a cluster shrinking as the bias increases from 2.4 to $4.0 \mathrm{~V}$. An ac component $(0.7 \mathrm{~V}$ square wave at $10 \mathrm{~Hz})$ is applied to further stabilize the crystal. The circulation period of the toroid is about $1.5 \mathrm{~s}$. (a) $0 \mathrm{~s}$. (b) $0.5 \mathrm{~s}$. (c) $1.0 \mathrm{~s}$. (d) $1.5 \mathrm{~s}$. (e) $2.0 \mathrm{~s}$. (f) $3.0 \mathrm{~s}$.

lation. Remarkably, the cluster's crystalline core is destroyed and reformed with each cycle. Neighboring clusters' oscillations do not become phase locked, nor do they necessarily pulsate at the same frequency. Indeed, oscillating clusters can coexist with steadily circulating or compactified clusters.

Within the range of biases for which flowerlike colloidal vortex rings reproducibly form (about 2.6-3.4 V), we also observe a variety of variant vortex ring structures that either coexist with or even replace the morphologies represented in Figs. 6 and 7. Some of these are shown in Fig. 10.

Figure 10(a) shows colloidal vortex rings whose coronas have sharply defined boundaries, rather than the diffuse coronas evident in Fig. 6(c). These clusters also sometimes form without a crystalline core as can be seen in Fig. 10(b),
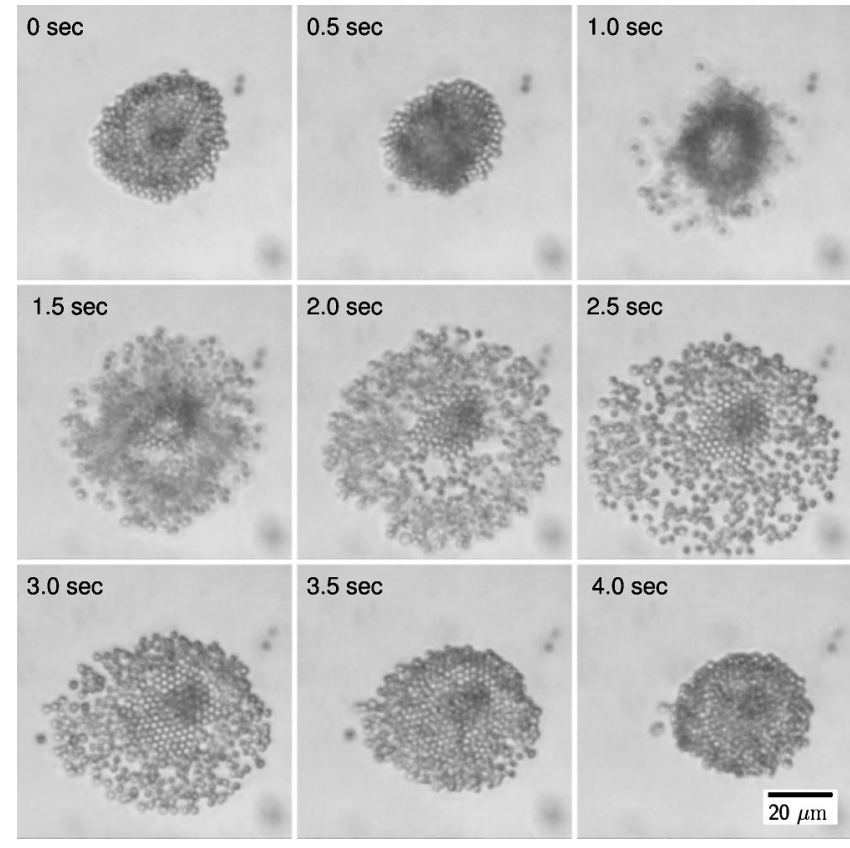

$4.0 \mathrm{sec}$

FIG. 9. This sequence of images, separated by $0.5 \mathrm{~s}$, shows one period of a vortex ring's breathing mode oscillation. $3.0 \mu \mathrm{m}$ silica spheres in $H$ $=200 \mu \mathrm{m}$ cell at $2.8 \mathrm{~V}$ constant bias. The cluster's core remains crystalline throughout the cycle. 


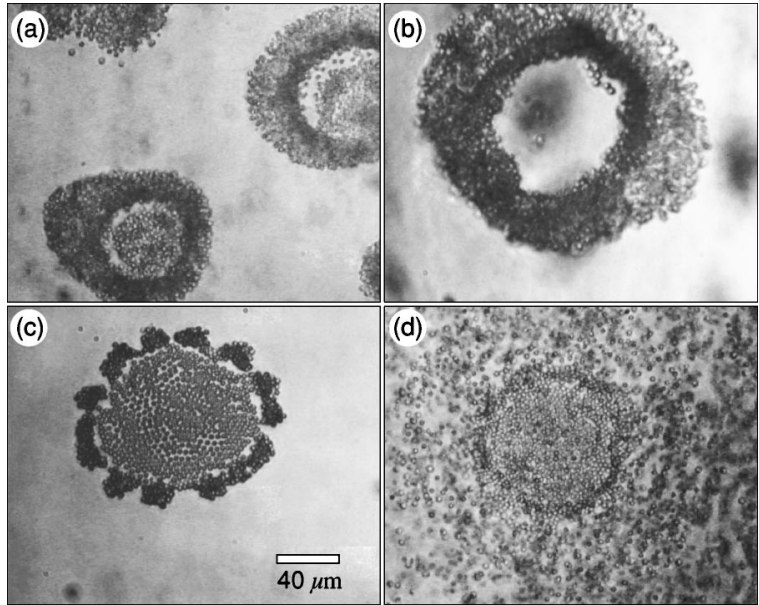

FIG. 10. Variations on the theme of colloidal vortex rings. $3.0 \mu \mathrm{m}$ diameter silica spheres in $H=200 \mu \mathrm{m}$ cells. (a) $3.0 \mathrm{~V}$, colloidal vortex rings with sharply defined coronas. (b) $2.6 \mathrm{~V}$, sharply bounded vortex ring without central core. (c) $2.6 \mathrm{~V}$, central crystal stabilized by a $0.1 \mathrm{~V}$ square wave at $1.0 \mathrm{~Hz}$ develops scalloped boundary. (d) $3.0 \mathrm{~V}$, rare variant in which the central core also is convective, $\phi \approx 150 \%$.

and the two types can coexist. Unlike the diffusely bounded clusters that undergo breathing mode instabilities, the corona in the dense toroidal morphology can undulate like a jellyfish. Figure 10(c) shows an extreme example of this circumferential instability.

In all these cases, the vigorously circulating vortex ring appears to be virtually decoupled from the central crystalline core. Figure 10(d) shows a relatively rare case in which some of the particles in the central core are swept into the vortex ring's circulation.

In roughly half of our experiments, we observed vortex rings coexisting with two-dimensional surface crystals ${ }^{17,18}$ that had nucleated on the upper electrode. The spheres in these crystals are driven to the upper electrode by the transient ionic current burst when the bias is initially applied. Particles that reach the upper electrode during these transients tend to stay there and subsequently form crystalline clusters through mechanisms that have been previously reported. ${ }^{17,18}$ Indeed, such surface crystals begin to form at biases below the onset for vortex ring formation. Above $2.6 \mathrm{~V}$, clusters hovering in the bulk coexist with crystals trapped on the upper surface. Decreasing the bias to $1.4 \mathrm{~V}$, which is the threshold for forming crystals on the upper electrode, causes all particles in crystals to sink to the bottom.

\section{Wormlike colloidal vortices}

Not all colloidal vortices adopt a ringlike configuration, as the examples shown in Fig. 11. Lower concentration suspensions tend to favor elongated vortex rings, such as those in Fig. 11(a), that can extend into linear wormlike structures such as those in Fig. 11(b). These clusters continue to circulate, but as linear rolls rather than vortex rings. As a result, wormlike colloidal vortices interact more strongly than colloidal vortex rings, and can organize themselves into largescale labyrinthine patterns, as in Fig. 11(c). As for the other patterns we have described, wormlike colloidal vortices are levitated to roughly $h=50 \mu \mathrm{m}$ in an $H=200 \mu \mathrm{m}$ cell.
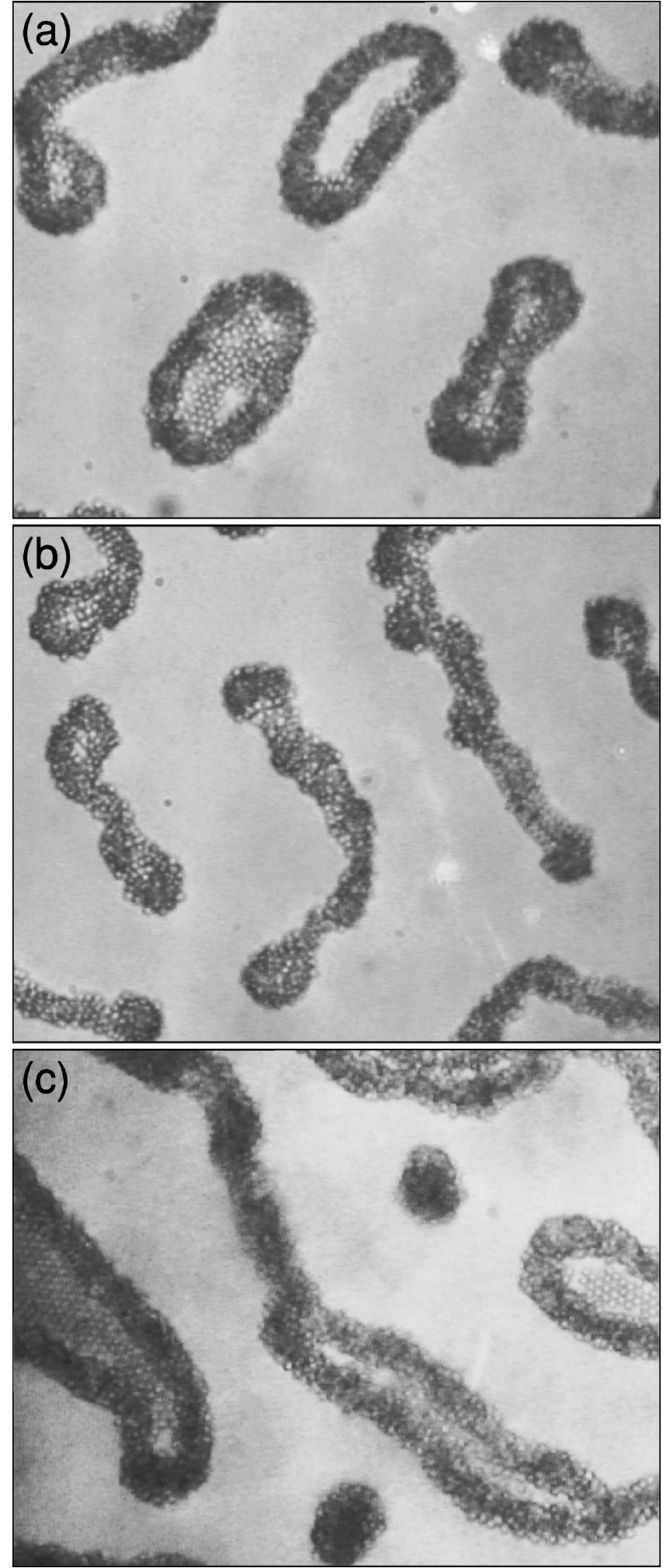

FIG. 11. Wormlike colloidal vortices. (a) $3.4 \mathrm{~V}$, elliptical colloidal vortex rings, both with and without crystalline cores, coexisting with wormlike colloidal vortices. (b) $3.4 \mathrm{~V}$, wormlike vortices extending from vortex rings. (c) $2.6 \mathrm{~V}$, labyrinth of wormlike vortices.

\section{Sedimented interfacial patterns}

Although the patterns described in the preceding section form reproducibly, they occasionally are replaced by qualitatively distinct labyrinthine and island-like patterns such as those shown in Fig. 12. Unlike the vigorously circulating 


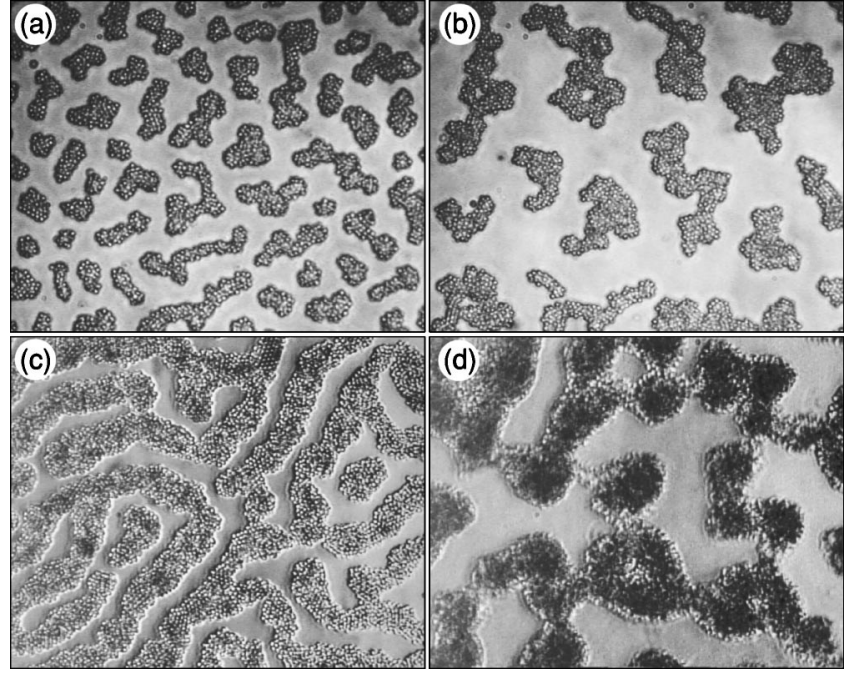

FIG. 12. Sedimented interfacial patterns. (a) Islands of $3.0 \mu \mathrm{m}$ diameter silica spheres in $H=90 \mu \mathrm{m}$ cell at $2.6 \mathrm{~V}$. (b) The same sample, after increasing the bias to $2.8 \mathrm{~V}$. (c) Labyrinths of $3.0 \mu \mathrm{m}$ diameter silica spheres in $H=200 \mu \mathrm{m}$ cell at $2.6 \mathrm{~V}$. (d) The same sample, after increasing the bias to $3 \mathrm{~V}$.

free-floating colloidal vortices, these slow-moving clusters form near the bottom electrode under the same conditions. These patterns also are distinct from the previously reported interfacial crystals, which form on the upper electrode under positive bias.

Once sedimented interfacial clusters nucleate, the choice between forming islands, as in Figs. 12(a) and 12(b), or labyrinths, such as Figs. 12(c) and 12(d), depends principally on the concentration of particles. Two-dimensional islands form at surface coverages of $10 \% \leqq \phi \lesssim 60 \%$ and twodimensional labyrinths at $60 \% \leqq \phi \lesssim 80 \%$. Threedimensional labyrinths form at higher concentrations. Islands and labyrinths can coexist at intermediate sphere concentrations.

The spheres in islands and labyrinths usually flow fluidly as the patterns form, in a manner that resembles the circulation in bulk colloidal vortices. Unlike bulk colloidal vortices, whose circulation retains its sense over time, labyrinthine domains sometimes flip circulation direction as they coarsen. Unlike Rayleigh-Bénard convection or bulk electroconvection, moreover, neighboring domains' circulation directions generally are not correlated. Larger labyrinthine domains, however, develop double-roll structures, with particles rising along the edges and sinking along the center line.

After a few minutes of circulation, islands and labyrinths often become jammed. Increasing the bias can fluidize them again, and coarsen their features. This can be seen in the transitions from Fig. 12(a) to Fig. 12(b) and from Fig. 12(c) to Fig. 12(d).

Increasing the bias still further causes coarsened blobs such as those in Fig. 12(d) to circulate faster and develop into interfacial toroidal vortex rings, an example of which appears in Fig. 13(a). These surface-hugging clusters differ from the free-floating vortex rings of the previous section in that they respond irreversibly to changes in bias. Reducing the bias on circulating blobs causes them to open out into structures resembling smoke rings, Fig. 13(b). This morphol-

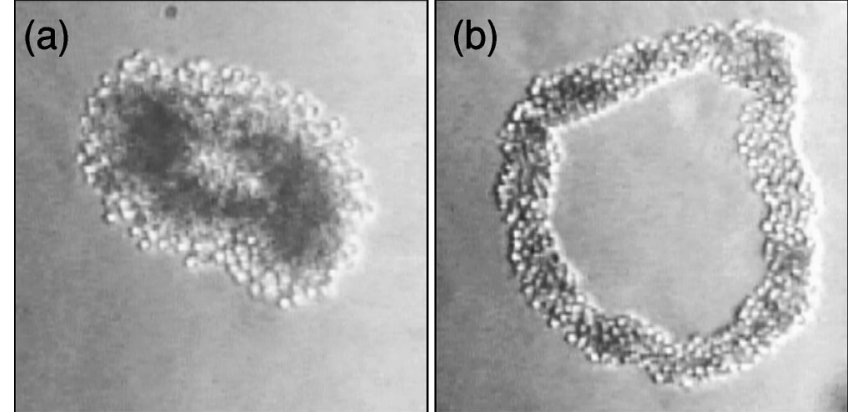

FIG. 13. Sedimented interfacial vortex opening out into a smoke ring cluster. $3.0 \mu \mathrm{m}$ spheres in an $H=200 \mu \mathrm{m}$ cell. (a) A typical vortex ring at $3.4 \mathrm{~V}$. (b) The same cluster at $2.8 \mathrm{~V}$.

ogy can be formed only by first increasing and then decreasing the bias, and clearly inherits its topology from the progenitor toroidal cluster.

The islands and labyrinths we have observed somewhat resemble the interfacial crystals described in Refs. 17 and 18, even though the electric field is reversed. We conjecture that gravity maintains the particles on the lower electrode, while the in-plane electrohydrodynamic attraction discovered in Refs. 17 and 18 draws them together into islands [Fig. 12(a)] and labyrinths [Fig. 12(c)]. Once formed, such close-packed clusters would have substantially reduced hydrodynamic drag coefficients, ${ }^{29}$ and so would remain sedimented at biases that would levitate a single sphere. Applying the bias rapidly appears to favor the formation of levitated clusters, presumably by disrupting this stabilizing mechanism, while slowly increasing the bias improves the chances to form interfacial clusters.

We have observed free-floating colloidal vortex rings coexisting with interfacial labyrinths and islands. This demonstrates that the two types of structures can be generated under the same conditions, and therefore are not distinguished by variations in the experimental procedure.

\section{E. Anomalous dynamics in relaxing colloidal vortices}

Turning off the driving bias after a pattern has formed frees the spheres to revert to their equilibrium field-free behavior. In most cases, particles sediment into a twodimensional Brownian fluid on the lower electrode. On other occasions, and particularly after prolonged runs at the upper range of biases, the sedimented particles no longer diffuse freely on the lower electrode but rather sit motionlessly where they land. This is the case for the particles in Fig. 14(A), which had been organized into a colloidal vortex ring before the field was switched off. These particles reached the lower electrode rapidly enough that their toroidal configuration is still clearly visible, and remained unchanged for several minutes. After this period, however, the particles began to diffuse normally, suggesting that they had not deposited onto the lower electrode. This conclusion is supported by the observation that a small positive bias suffices to levitate the motionless spheres, as shown in Fig. 14(B). Once the positive bias removed, however, the particles sediment again to the lower electrode and form small crystals as they settle, a typical result appearing in Fig. 14(C). The spheres in these 


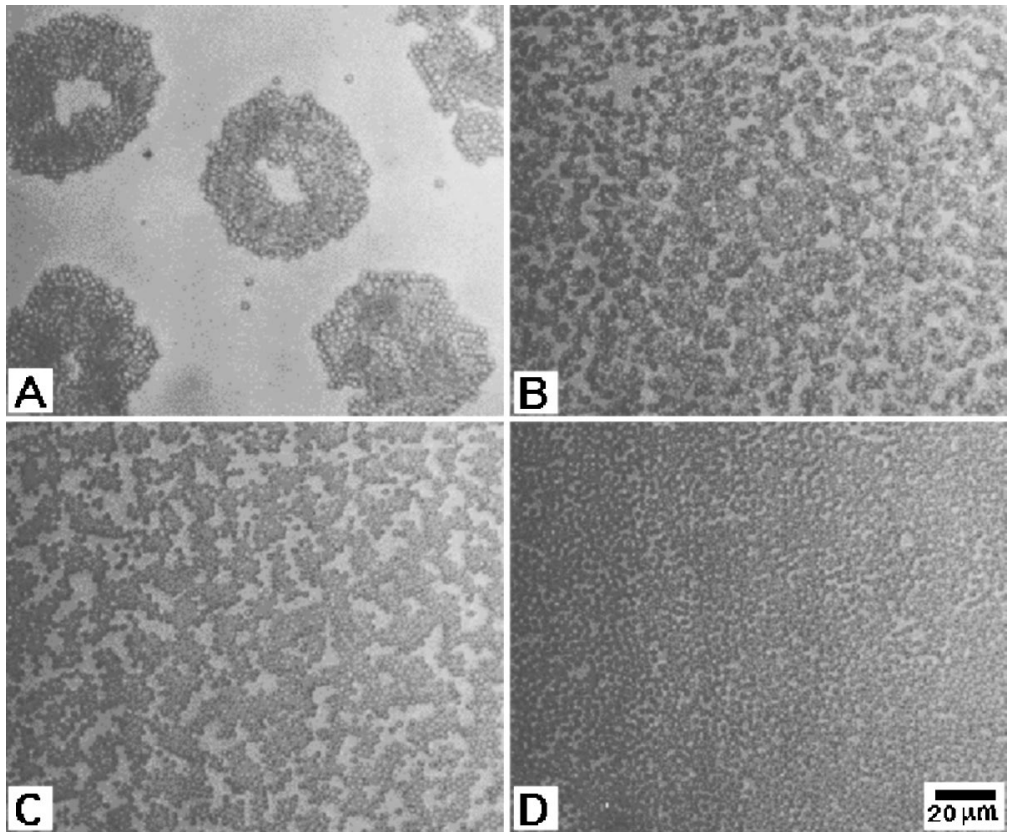

FIG. 14. Relaxation behavior of toroidal vortex rings of $3 \mu \mathrm{m}$ silica spheres after a $4 \mathrm{~V}$ bias is removed. (A) $0 \mathrm{~V}$, particles sedimented onto the lower electrode retain the basic profile of their cluster's toroidal geometry. Left in this state, these particles would require several minutes to relax to their normal diffusive state. (B) Applying a positive bias of $0.8 \mathrm{~V}$ levitates the particles slightly and allows them to relax to a uniform density. (C) Returning to $0 \mathrm{~V}$ eliminates any diffusive motion and causes the spheres to form sedimented crystalline domains. (D) After $3 \mathrm{~min}$, the spheres begin to diffuse and relax to a homogeneously random state.

self-organized crystals still display no Brownian motion for several minutes, after which they revert to their equilibrium behavior, as shown in Fig. 14(D). This bizarre transient behavior appears not to have been described before, and its origin is unknown.

\section{F. Dependence on control parameters}

Similar patterns and trends emerge if the bias voltage is applied abruptly or increased gradually enough that diffuse tumbling clouds form before coalescing into stable clusters. The typical number of particles in a steady-state cluster tends to increase with the bias. Slightly changing the bias once clusters have formed usually does not affect this number because merging and splitting clusters to achieve the new optimal cluster size imposes a substantial kinetic barrier. Large bias changes, however, can break up all the clusters and reorganize them into clusters with the appropriate number of particles. For example, groups of three clusters can merge then split into cluster pairs with an increase in bias. Reducing the applied bias reverses the sequence of transitions, without obvious hysteresis.

The interelectrode spacing also does not affect electrokinetic pattern formation over quite a large range, with similar patterns appearing in sample cells at $H=90,200$, and $390 \mu \mathrm{m}$, all with similar crossover voltages. Bulk clusters are always observed to float at about $h=H / 4$, as shown in Fig. 15. Interfacial patterns form at a fraction of this height. Patterns do not form in thinner cells, however, for example, we observed that particles in a cell $H=40 \mu \mathrm{m}$ thick were pushed directly to the upper electrode and formed no bulk clusters.

Adding salt slows the particles' motions and favors the formation of tumbling clouds instead of quasistationary clusters. Patterns are blurred in $0.1 \mathrm{mM} \mathrm{NaCl}$ solution, and disappear altogether in $0.5 \mathrm{mM}$ solution. For example, $3.0 \mu \mathrm{m}$ silica spheres dispersed in $0.2 \mathrm{mM} \mathrm{NaCl}$ solution failed to form any quasistationary structures, but rather entered an ir- regular cycle of collapsing into concentrated domains and then bursting like fireworks as shown in Fig. 16.

Unlike the previously reported interfacial crystals, which form for a wide variety of particle sizes and compositions, the rich panoply of patterns we have described appears only for particles meeting specific criteria of size and weight. To clarify this, we compared the electrohydrodynamic pattern formation behavior in charge-stabilized dispersions of colloidal silica spheres, PS spheres and brominated PS spheres whose densities are $1.96,1.05$, and $1.6 \mathrm{~g} / \mathrm{cm}^{3}$, respectively. The surface charge densities of PS and brominated PS are similar and do not depend on the $p \mathrm{H}$. Both are more highly charged than the silica particles we have discussed so far. Silica's charge density, moreover, varies with $p \mathrm{H}$, with an isoelectric point at $p \mathrm{H} 2$.

The behavior of $2.9 \mu \mathrm{m}$ diameter silica spheres is very similar to that of the $3.0 \mu \mathrm{m}$ diameter spheres we have discussed to this point. These also can form various bulk and low-floating patterns. Substantially larger silica spheres never leave the lower electrode at biases below the threshold for bulk electroconvection. Substantially smaller, lighter, and more diffusive silica spheres create less well organized pat-

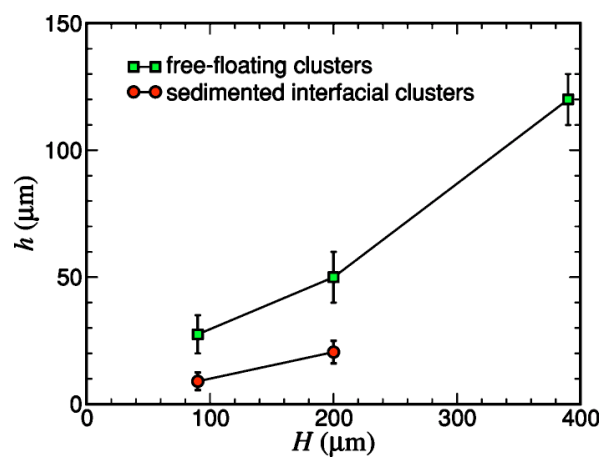

FIG. 15. Heights of free-floating and sedimented interfacial patterns. The error bars represent the variations from run to run. In each experiment, all clusters float at the same height, with variations well within the error bars. 


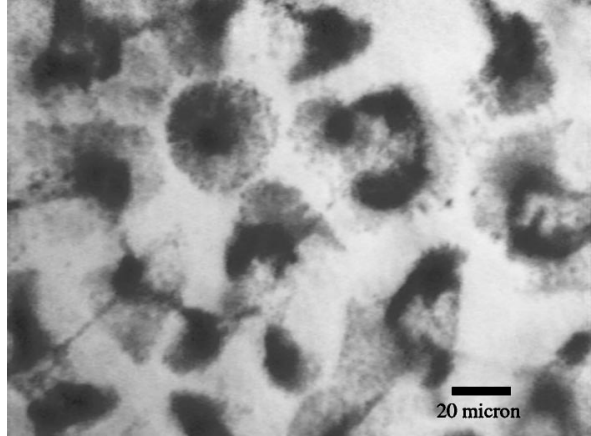

FIG. 16. $3.0 \mu \mathrm{m}$ diameter silica spheres in $0.2 \mathrm{mM} \mathrm{NaCl}$ solution. $H$ $=200 \mu \mathrm{m} 4 \mathrm{~V}$ bias. Particles repeatedly collapse into dense clusters and then burst out like fireworks.

terns. For example, $1.58 \mu \mathrm{m}$ silica particles usually only form unstable tumbling clouds at comparatively low biases before being pushed to the upper electrode at higher biases.

From previous studies, ${ }^{18}$ it is known that electrokinetic interactions are weaker for smaller spheres. This would tend to reduce the forces binding patterns such as colloidal vortex rings together. The smaller spheres' more vigorous diffusion also would tend to hinder the localized clusters' formation. It also suggests a possible role for gravity, which can be highlighted by considering spheres comparable in size to the pattern-forming silica particles that are composed of different materials.

Unlike silica spheres, the nearly neutrally buoyant PS spheres fail to form any bulk patterns at any accessible bias over the entire diameter range from 0.3 to $7.0 \mu \mathrm{m}$. These highly charged spheres should have comparably strong fieldinduced interactions to their silica counterparts. Their diffusivity also should be similar. Their failure to form bulk patterns demonstrates that the gravity plays a central role in the pattern formation we have described, a conclusion consistent with the observation that no bulk patterns form at negative bias.

Dense brominated PS spheres $1.6 \mu \mathrm{m}$ in diameter can form tumbling clouds similar to those formed by comparably sized silica spheres. Whereas clouds of $1.58 \mu \mathrm{m}$ silica spheres tumble vigorously, however, clouds of brominated PS particles have no detectable internal convection. Such quiescent itinerant clouds also appear for dispersions of $3 \mu \mathrm{m}$ silica spheres but only at much lower concentrations. In both cases, each drifting cloud is about $50 \mu \mathrm{m}$ across and its constituent particles are separated by about $10 \mu \mathrm{m}$.

Stable clusters also do not form at very high or very low particle concentrations. Dispersions at $\phi \gtrsim 200 \%$ form chaotic clouds at low bias and jammed clusters at high bias. The absence of cyclic motion in low-concentration dispersions demonstrates that the flow patterns we have observed do not arise in the electrolyte alone under the relevant range of conditions, but rather require the spheres' presence. The patterns' concentration dependence further highlights the role of cooperative electrohydrodynamic interactions in establishing the distinct patterns. Isolated particles' behavior provides insights into the forces that help to clarify how such patterns form.

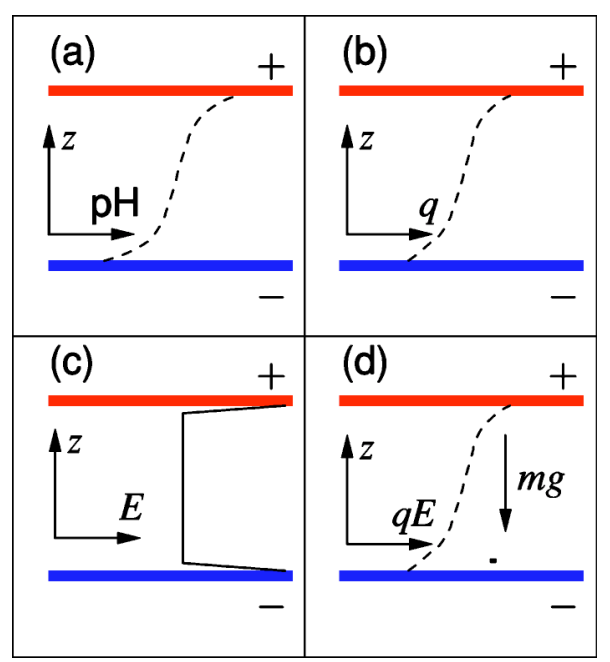

FIG. 17. Schematic representation of the gravitational and electrostatic forces on a silica sphere. (a) Dissociation of water during hydrolysis establishes a vertical $p \mathrm{H}$ gradient. (b) The charge $q$ on a sphere tracks variations in $p \mathrm{H}$. (c) The electric field $E$ is nonvanishing but substantially independent of height outside of the Debye-Hückel screening layers near the electrodes. (d) Even so, the charge-regulating spheres experience an upward force that increases with height in the cell. This force opposes the force due to gravity.

\section{G. Single-particle behavior}

To best highlight the role of collective behavior in electrohydrodynamic pattern formation, we studied the simplest case: a single silica sphere in an electrolysis cell. A single charged colloidal sphere of buoyant mass $m$ and charge $q$ in a constant electric field not only experiences a downward gravitational force $m g$ and an electrostatic force $q \mathbf{E}$ but also more complicated electrohydrodynamic and osmotic forces due to the electrolyte's response to the field. When the lower electrode is negative, the positive screening cloud of the sphere flows downward. These flowing ions, including $\mathrm{OH}^{-}$ and $\mathrm{H}_{3} \mathrm{O}^{+}$created by hydrolysis, entrain a flow of water. The friction between this electrohydrodynamic flow and the sphere drags the sphere downward. The sphere's cloud of screening ions is deformed not only by the electric field but also by the hydronium flux. This downward osmotic force and the downward electrohydrodynamic drag are secondorder responses to the electric field, and therefore should be smaller than the electrostatic levitating force $q \mathbf{E}$.

Straightforward considerations allow us to estimate the bias needed to levitate a particle against gravity. A single sphere sediments through water at speed $v_{g}=m g b=\sigma^{2}$ $\left(\rho-\rho_{0}\right) g /(18 \eta)$, where $\rho_{0}=1 \mathrm{gm} / \mathrm{cm}^{3}$ is the density of water and $\eta=1 \mathrm{cP}$ is its viscosity. For $3 \mu \mathrm{m}$ diameter silica spheres, $v_{g} \approx 5 \mu \mathrm{m} / \mathrm{s}$. The same particle's electrophoretic velocity saturates at $v_{e} \approx e_{0} \Sigma\left(V-V_{t}\right) /(H \kappa \eta)$, where $\kappa^{-1}$ is the electrolyte's Debye-Hückel screening length, and $e_{0}$ is the proton charge. ${ }^{1}$ If we choose the surface charge density $\Sigma_{p \mathrm{H}=5} \sim 5000 \mu \mathrm{m}^{-2}$ (Ref. 23) and $\kappa^{-1}=0.1 \mu \mathrm{m}$, these two velocity scales would be equal at about $V-V_{t}=0.1 \mathrm{~V}$. Thus, considering the neglected electrohydrodynamic and osmotic drags, the threshold for levitation should be at least $0.1 \mathrm{~V}$ higher than the water decomposition voltage. This is consistent with our experiments.

In fact, the $p \mathrm{H}$ is not uniform across the cell. Figure 17 


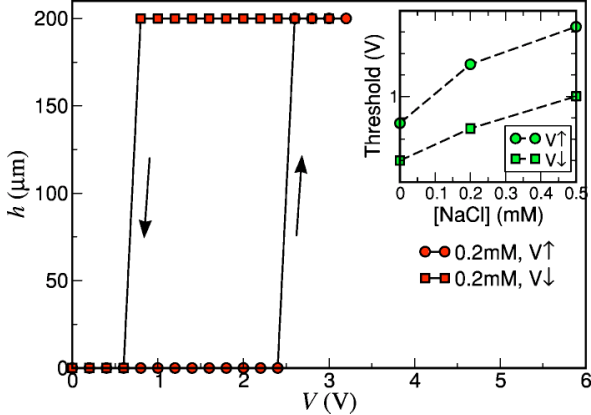

FIG. 18. Height attained by a single $3 \mu \mathrm{m}$ diameter in a $H=200 \mu \mathrm{m}$ cell as a function of applied bias. The voltage is increased and then decreased step by step. Inset, the threshold voltage required to levitate spheres.

schematically shows how the profiles of $p \mathrm{H}$, sphere charge $q$, electric field $E$, and electric force $q E$ vary with height in the cell. Even though the electric field is uniform in the bulk, the electrokinetic force levitating a charge-regulating silica sphere increases with height. When this force competes with gravity, therefore, it is possible to obtain two equilibrium heights, one near the lower electrode when gravity dominates, and another near the upper electrode where the electrokinetic force dominates.

The situation is more complicated for two spheres because hydrodynamic forces between the spheres and bounding surfaces are long ranged. Reference 28 shows the numerically calculated streamlines for one or two electrophoretically levitated charged spheres. Two particles sinking towards the lower electrode create a back flow which tends to drive them apart. ${ }^{30}$ Conversely, two electrically levitated particles will attract each other. This electrohydrodynamic interaction at least heuristically explains why an initially homogeneous colloidal fluid breaks up into discrete clusters as it is driven away from the lower electrode.

We observed that the threshold for levitating a single sphere is lower than that for levitating an entire monolayer. This most likely reflects the reduced hydrodynamic drag coefficient for many spheres in a monolayer, as compared to that for a single sphere. ${ }^{29}$ The increased hydrodynamic attraction generated by the intensified ionic fluxes in the more concentrated dispersions would tend to draw these sedimented spheres together into clusters. This, however, tends to reduce their overall hydrodynamic drag, ${ }^{29}$ causing them to rise less than they otherwise might have.

To compare our system's behavior with these predictions, we performed experiments on $3 \mu \mathrm{m}$ diameter silica spheres from a monolayer with areal density below $10^{-4} \mu \mathrm{m}^{-2}$, i.e., fewer than three particles per frame. Abruptly applying a bias causes these isolated spheres to jump off the lower electrode during the transient in which vertical ionic concentration gradients are established. Below a threshold voltage, the particles eventually return to the lower wall. Above this threshold, they rise to the top wall and remain suspended. Figure 18 shows how the single sphere's height changes with applied voltage as the voltage is first increased and then decreased. The point at which a single sphere is electrolevitated to the upper wall is hysteretic: levitated particles only return to the lower wall when the voltage is lowered substantially. The explanation for this hysteresis also can be found in Fig. 17. At the critical bias, $|q \mathbf{E}(z)| \gtrsim m g$ for all $z$ in the bulk. By contrast, a particle can only fall from the upper electrode, when the field falls below threshold at the upper electrode $|q \mathbf{E}(H)| \lesssim m g$. Therefore, the two thresholds are different. The inset to Fig. 18 shows this hysteresis more clearly at three different salt concentrations. Such hysteresis supports the contention that the levitating force acting on silica spheres increases with the height $h$.

For salt concentration above $1 \mathrm{mM}$, a single particle can never be forced to the upper electrode. This is consistent with the observation that particles move less vigorously and cooperative structures are harder to form at high ionic concentrations.

Attractions between particles are detectable only at relatively low particle concentrations (e.g., $\phi \simeq 10^{-4}$ ) and high applied voltages (e.g., $2 \mathrm{~V}$ ). In such cases, particles attract each other as they levitate and so form tumbling clouds. The typical interparticle separation in the most diffuse clouds is about $10 \mu \mathrm{m}$ and decreases with increasing bias. These fieldinduced attractions are surprisingly long-ranged, extending to roughly $50 \mu \mathrm{m}$. At biases above roughly $5 \mathrm{~V}$, even isolated spheres travel in circular orbits whose diameters range from 50 to $150 \mu \mathrm{m}$ in the vertical plane. This motion is due to electroconvection in the underlying electrolyte. It almost certainly plays no role in forming the highly organized and substantially smaller-scale patterns discussed above.

Generally speaking, single particles are not stably levitated into the bulk of the electrolyte, and they certainly do not trace out the complex trajectories characterizing microscopic patterns such as those in Figs. 6-16. These observations help to confirm that such patterns do not arise from electroconvection in the electrolyte alone. We are left to conclude that the spheres play an active role in dynamical pattern selection.

\section{DISCUSSION}

We have demonstrated that many distinctive patterns reproducibly emerge from the interplay of gravity and electrohydrodynamic forces in charge-stabilized colloid. At biases above the threshold for hydrolysis but below the onset of electroconvection, only fluxes of $\mathrm{H}_{3} \mathrm{O}^{+}$and $\mathrm{OH}^{-}$ions flow through the system. Electroneutrality favors overlap of these fluxes, however, "friction" between opposite flows is minimized if these flows are separated. The optimal balance between these effects separates the fluxes by a characteristic lengthscale comparable to the Debye-Hückel screening length, or roughly $0.1 \mu \mathrm{m}$. This is much smaller than the extent of the experimentally observed clusters whose diameters extend to roughly $100 \mu \mathrm{m}$. Electroneutrality suppresses the formation of such large-scale convective structures in the absence of the charged spheres. In other words, particles are not simply passive tracers for a preexisting flow in the electrolyte but rather they and their charges are essential for establishing the convective flows at the heart of the observed patterns.

There are three general features about the dynamics of all clusters. (1) They have the same circulating direction as 
shown in Fig. 1. (2) The circulation speed increases with the bias as clusters tend to become more compact. (3) The number of particles in each cluster increases with the bias. The bias-induced attraction of the second feature is crucial to understand the clusters' formation. The breathing mode in Figs. 9 and 16 may result from competition between this as-yet unexplained attraction and a combination of screenedCoulomb repulsions and induced dipole repulsions among spheres.

While the sequence of dynamical transitions from sedimented layer to tumbling clouds to highly organized clusters is reproducible, the crossover potentials depend sensitively on the changing electrochemical properties of the electrodes, and therefore can vary by as much as $\pm 0.4 \mathrm{~V}$ from run to run.

\section{SUMMARY}

We have reported a large family of previously unrecognized self-organized colloidal patterns that form in constant vertical electric fields at biases just above the decomposition voltage of water. Our simple electrolysis cell gives rise to a complicated coupled system of hydrodynamic flows and ionic fluxes that are shepherded by the very colloidal particles they transport. We have discussed the electrohydrodynamic forces on a single particle in this system, and suggested how many-body coupling might give rise to the various dynamical structures we have observed. At low voltages, colloidal spheres in a specific range of charges, densities, and mobilities are found to form various microscopic quasi-steady-state clusters through the competition of gravity and electrohydrodynamic levitating forces. These patterns fall into two categories: clusters levitated into the bulk and clusters localized near the lower electrode. Their structures and evolutions are different, but all share some general features, such as persistent circulation and coarsening with increasing voltage. The simple behavior of a single particle in the field demonstrates that these microscopic clusters are formed cooperatively. Patterns' dependence on such control parameters as the composition and number density of particles, cell thickness $H$, salt concentration, and applied voltage range have been discussed.

\section{ACKNOWLEDGMENT}

This work was supported by the Donors of the Petroleum Research Fund of the American Chemical Society.

${ }^{1}$ W. B. Russel, D. A. Saville, and W. R. Schowalter, Colloidal Dispersions, Cambridge Monographs on Mechanics and Applied Mathematics (Cambridge University Press, Cambridge, 1989).

${ }^{2}$ E. Muth, Kolloid-Z. 41, 97 (1927).

${ }^{3}$ W. M. Winslow, J. Appl. Phys. 20, 1137 (1949).

${ }^{4}$ B. R. Jennings and M. Stankiewicz, Proc. R. Soc. London, Ser. A 427, $321(1990)$

${ }^{5}$ Y. Hu, J. L. Glass, A. E. Griffith, and S. Fraden, J. Chem. Phys. 100, 4674 (1994).

${ }^{6}$ H. Isambert, A. Ajdari, J. L. Viovy, and J. Prost, Phys. Rev. Lett. 78, 971 (1997).

${ }^{7}$ H. Isambert, A. Ajdari, J.-L. Viovy, and J. Prost, Phys. Rev. E 56, 5688 (1997).

${ }^{8}$ A. E. Larsen, Ph.D. thesis, The University of Chicago, Chicago, IL, 1996.

${ }^{9}$ A. E. Larsen and D. G. Grier, Phys. Rev. Lett. 76, 3862 (1996).

${ }^{10}$ A. E. Larsen and D. G. Grier, Nature (London) 385, 230 (1997).

${ }^{11}$ P. Richetti, J. Prost, and P. Barois, J. Phys. (France) Lett. 45, L1137 (1984).

${ }^{12}$ Q. H. Wei, X. H. Liu, C. H. Zhou, and N. B. Ming, Phys. Rev. E 48, 2786 (1993).

${ }^{13}$ X. Y. Lei et al., Phys. Rev. E 52, 5161 (1995).

${ }^{14}$ Q. H. Wei, X. Y. Lei, C. H. Zhou, and N. B. Ming, Phys. Rev. E 51, 1586 (1995).

${ }^{15}$ W. D. Ristenpart, I. A. Aksay, and D. A. Saville, Phys. Rev. Lett. 90, 128303 (2003).

${ }^{16}$ A. Yethiraj and A. Blaaderen, Nature (London) 421, 513 (2003).

${ }^{17}$ M. Trau, D. A. Saville, and I. A. Aksay, Science 272, 706 (1996).

${ }^{18}$ M. Trau, D. A. Saville, and I. A. Aksay, Langmuir 13, 6375 (1997).

${ }^{19}$ M. Böhmer, Langmuir 12, 5747 (1996).

${ }^{20}$ Y. Solomentsev, M. Böhmer, and J. L. Anderson, Langmuir 13, 6058 (1997).

${ }^{21}$ X. D. Duan and W. L. Luo, Int. J. Mod. Phys. B 15, 837 (2001).

${ }^{22}$ Y. Han and G. G. Grier, Nature (London) 424, 267 (2003).

${ }^{23}$ S. H. Behrens and D. G. Grier, J. Chem. Phys. 115, 6716 (2001).

${ }^{24}$ J. O. Bockris and A. K. N. Reddy, Modern Electrochemistry (Plenum, New York, 1970).

${ }^{25}$ G. Kortüm, Treatise on Electrochemistry (Elsevier, Amsterdam, 1965).

${ }^{26}$ F. Nadal, F. Argoul, P. Hanusse, B. Pouligny, and A. Ajdari, Phys. Rev. E 65, 061409 (2002).

${ }^{27}$ K. Shariff and A. Leonard, Annu. Rev. Fluid Mech. 24, 235 (1992).

${ }^{28}$ T. M. Squires, J. Fluid Mech. 443, 403 (2001).

${ }^{29}$ J. Happel and H. Brenner, Low Reynolds Number Hydrodynamics (Kluwer, Dordrecht, 1991).

${ }^{30}$ T. M. Squires and M. P. Brenner, Phys. Rev. Lett. 85, 4976 (2000). 